\title{
ANÁLISE DE GLICOCORTICOSTERÓIDES POR CG-EM: UMA NOVA ABORDAGEM DE DERIVATIZAÇÃO PARA O CONTROLE DE DOPAGEM NO ESPORTE
}

\author{
Henrique M. G. Pereira*, Marlice A. S. Marques, Jarí N. Cardoso e Francisco R. Aquino Neto \\ Instituto de Química, Universidade Federal do Rio de Janeiro, Cidade Universitária, 21949-900 Rio de Janeiro - RJ
}

Recebido em 30/10/01; aceito em 28/3/02

\begin{abstract}
ANALISYS OF GLUCOCORTICOSTEROIDS BY GC-MS: A NEW APPROACH OF DERIVATIZATION STEP FOR DOPING CONTROL IN SPORT. Glucocorticosteroids (CT) show a powerful anti-inflammatory effect. In view of this, they are extensively used in sports medicine, but their misuse (systemic administration) can lead to severe injuries on athletes and has been restricted by the IOC in recent years. For GC-MS analysis, a derivatization step is necessary in order to convert them to a stable form, thus preventing thermal breakdown with the loss of the ketone side chain. Derivatization of CT is reviewed and a new approach of silylation is presented for screening of $\mathrm{CT}$ in urine.
\end{abstract}

Keywords: glucocorticosteroids; GC-MS; doping control.

\section{INTRODUÇÃO}

Glicocorticosteróides (CT) são esteróides polifuncionais secretados pela supra-renal que apresentam como funções principais a homeostasia de eletrólitos como o íon sódio $\left(\mathrm{Na}^{+}\right)$e potássio $\left(\mathrm{K}^{+}\right)$, a mediação no metabolismo de carboidratos, proteínas e lipídios e, principalmente, uma potente ação antiinflamatória ${ }^{1}$. Esta ampla gama de efeitos gerada pela administração de CT endógenos e sintéticos resulta em uma série de aplicações terapêuticas que vão do tratamento da artrite até a terapêutica de neoplasias malignas ${ }^{1,2}$. A Figura 1 esquematiza a biossíntese dos esteróides endógenos ${ }^{1-3}$.

A principal utilização de CT em desacordo com a sua destinação médica está relacionada à dopagem esportiva, tanto em humanos como em animais (especialmente cavalos de corrida) ${ }^{4,5}$. A principal razão para a utilização de $\mathrm{CT}$ está relacionada à sua ação antiinflamatória e analgésica ${ }^{6,7}$. A utilização de CT permite a participação de atletas (e animais) com lesões articulares em provas esportivas reprimindo provisoriamente a dor e a inflamação. Disto resultam conseqüências gravíssimas para o atleta, freqüentemente irreversíveis.

Pelo exposto, o Comitê Olímpico Internacional (COI) restringe a utilização sistêmica de CT. Deste modo, administrações pelas vias oral, retal, intravenosa e intramuscular ficam proibidas. Administrações intra-articulares e tópicas são permitidas, desde que previamente declaradas por um médico responsável ${ }^{8,9}$.

Ao longo das últimas duas décadas, técnicas analíticas como a cromatografia líquida com detector de ultravioleta (CLAE-UV) ${ }^{10,11}$, cromatografia líquida acoplada à espectrometria de massas (CLAE$E M)^{4,11-13}$ e cromatografia gasosa acoplada à espectrometria de massas com ionização negativa (CG-EM-IN $)^{5-7,14}$ e cromatografia gasosa acoplada à espectrometria de massas por ionização positiva (CG$\mathrm{EM})^{15-27}$ têm sido empregadas na detecção de esteróides polifuncionais.

A técnica de CLAE-UV apresenta como maiores limitações a baixa sensibilidade e a baixa especificidade devido à falta de informação estrutural sobre o analito de interesse. Tanto a CLAE-EM como a CG-EM-IN têm merecido grande destaque por sua alta sensibilidade. Entretanto, o emprego de tais técnicas de triagem em la-

*e-mail: ladetec@iq.ufrj.br boratórios de controle de dopagem se torna crítico, uma vez que são exigidos, entre outros requisitos, grande reprodutibilidade e praticidade de manuseio devido ao elevado número de amostras.

Deste modo, a CG-EM tem sido a técnica analítica mais utilizada dentro do cotidiano de laboratórios de controle de dopagem na análise de CT e outros esteróides.

Estruturalmente, CT apresentam grupos polares, hidroxilas ou cetonas conjugadas, na posição 3 (anel A) e uma cadeia lateral hidroxicetônica na posição 17 (anel D). Grupos cetona ou hidroxila são também comuns na posição 11 (anel C), (Figuras 2a e 2b). Alguns CT sintéticos apresentam substituições na posição 16 , como a dexamentasona $(\mathrm{R}=\mathrm{OH})$ e a triancinolona $\left(\mathrm{R}=\mathrm{CH}_{3}\right)$ (Figura $\left.2 \mathrm{c}\right)$. $\mathrm{O}$ metabolismo de CT envolve, normalmente, a hidroxilação da posição 6, como no 6 $\beta$-hidroxicortisol e a redução dos grupamentos cetona presentes na posição 3 , como no tetraidrocortisol e na tetraidrocortisona).

\section{Análise de glicocorticosteróides por CG}

O emprego da técnica de cromatografia gasosa (CG) na análise de CT preconiza a adoção de uma etapa de derivatização na preparação da amostra, uma vez que a ramificação hidroxicetônica na posição 17 torna os analitos passíveis de termodegradação ${ }^{16}$.

Os primeiros estudos de derivatização de esteróides datam do início da década de $1960^{28}$ (os primeiros estudos de derivatização especificamente sobre CT foram gerados apenas em meados da década seguinte $\left.{ }^{16}\right)$. A presença de grupamentos hidroxila e cetona sugeriram o emprego de derivados trimetilsilila, inicialmente através de um mecanismo de catálise ácida com trimetilbromosilano ${ }^{17}$. Entretanto, observou-se diferentes razões de reatividade de acordo com o ambiente químico dos grupos cetonas, com formação de poliderivados e conseqüente queda de rendimento.

Neste contexto, Fales e Luukainen ${ }^{18}$ utilizaram a clássica reação de formação de metoximas (MO derivados) para a proteção das carbonilas de cetoesteróides (Figura 3).

Os bons resultados observados conduziram Fales ${ }^{18}$ a recomendar o uso desses derivados na análise estrutural de cetoesteróides por CG-EM. Porém, tal procedimento, como esperado, não protegia os grupamentos $\mathrm{OH}$ presentes.

A presença de grupos hidroxila em conjunto com carbonilas 


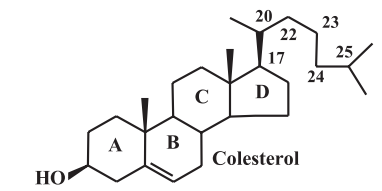

$$
\begin{array}{lll}
\text { (1): } 20 \text {-22-desmolase } & \text { (5) } 17 \beta \text {-HSD } & \text { (9) } \mathbf{P 4 5 0}_{21} \\
\text { (2): } 3 \beta \text {-HSB } / \Delta \text { 5-4-isomerase } & \text { (6): Aromatase } & \text { (10) } \mathrm{P}^{2} 0_{11} \\
\text { (3): } 17 \alpha \text {-hidroxilase } & \text { (7); } 5 \alpha \text {-redutase } & \\
\text { (4): } 17-20 \text {-desmolase } & \text { (8): } 3 \alpha \text {-HSD } & \text { (11) } P 450
\end{array}
$$

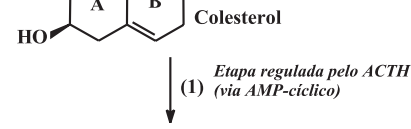<smiles>CC(=O)C1CCC2[C@H]3CC(C)=C4CC(O)CCC4(C)C3CC[C@]12C</smiles><smiles>CC(=O)[C@H]1CCC2C3CCC4(C)CCC(=O)C=C4C3CC[C@]21C</smiles>

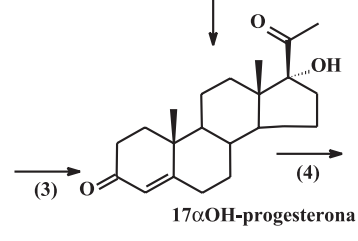<smiles>C1CCCC1</smiles>
DHEA-S

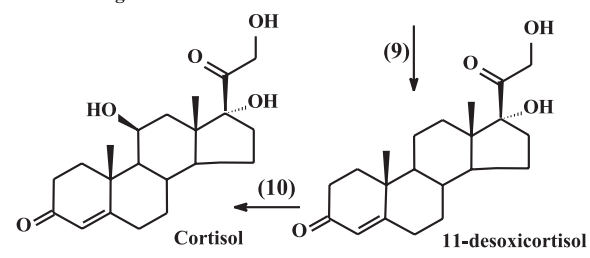<smiles>C#CCC1CCC(=O)C2(C)CCCC1C21CCc2cc(O)ccc21</smiles>

Estrona<smiles>C#CC1C=C2CCC3C(CCC4CCCC43C)C2CC1</smiles><smiles>CCCC1CCC2(C)CC(=O)CCC2(C)C1CCC1(C)CCCC1O</smiles>

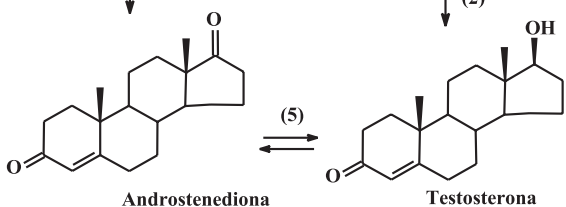

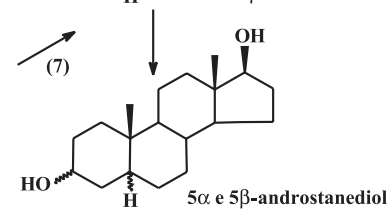

Figura 1. Biossíntese e metabolismo dos esteróides endógenos ${ }^{2-3}$

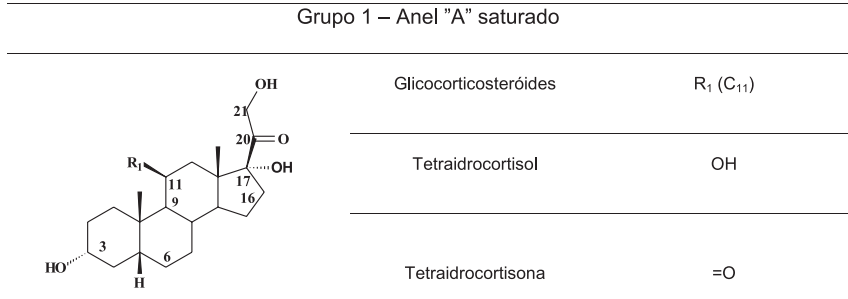

Figura 2a. CT avaliados com anel A saturado

Grupo 2 - Anel "A" 4,5-eno

Figura 2b. CT avaliados com estrutura 3-ceto-4,5-eno

\begin{tabular}{cccccc}
\hline \multicolumn{7}{c}{ Grupo 3 - Anel "A" 1,2 / 4,5-dieno } \\
\hline
\end{tabular}

Figura 2c. CT avaliados com estrutura 1,2 / 4,5-dieno

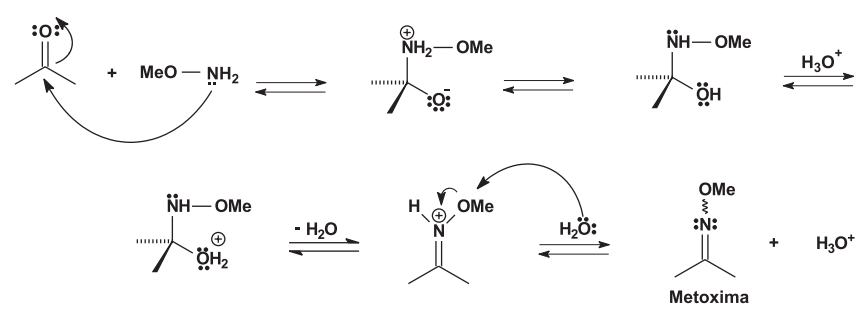

Figura 3. Reação de formação de metoximas ${ }^{18}$ 
cetônicas levaram Horning e colaboradores ${ }^{28}$ a adotar uma reação em duas etapas. A primeira envolvendo a reação dos CT com hidrocloreto de metoxiamina. A segunda etapa consistindo da reação da metoxima formada com N-N-bis-trimetilsililacetamida (BSA) e trimetilclorossilano (TMCS) à temperatura ambiente por até cinco horas, gerando assim derivados metoximatrimetrilsilila (MO-TMS). A adoção de duas etapas de derivatização apresentou como vantagem a eliminação da formação de multiderivados através da proteção dos grupos cetonas e posterior silanização dos grupos hidroxila. Além disso, o emprego dos derivados MO-TMS mostrou-se conveniente devido à sua estabilidade térmica. Entretanto, apesar da derivatização total dos grupos polares no carbono 3, ficou demonstrada a possibilidade de formação de isômeros geométricos sin e anti da metoxima nessa posição, o que para análise quantitativa é considerado uma desvantagem (Figura 4). Não se observa isomerização na formação da metoxima na posição 20 .

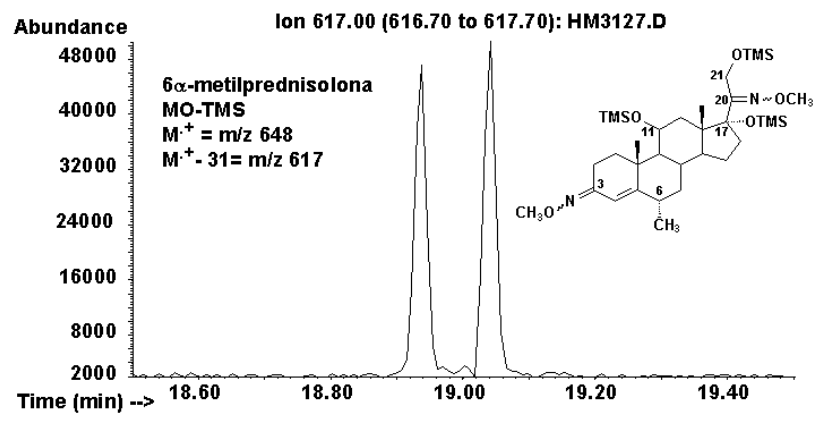

Figura 4. Cromatogramas dos isômeros geométricos $\sin e$ anti na formação de metoxima nas posições 3 na $6 \alpha$-metilprednisolona $(\mathrm{m} / \mathrm{z}$ 617)

Em 1969, Chambaz e Horning ${ }^{19}$ desenvolveram estudos sistemáticos de silanização com hidroxicetoesteróides, através de reações não catalisadas e catalisadas com o objetivo de avaliar os graus de impedimento dos grupos hidroxilas, em relação à sua posição na molécula. Na avaliação da androsterona, a cetona em C17 foi previamente protegida pela formação da metoxima, o que possibilitou a formação de apenas um derivado (Figura 5).

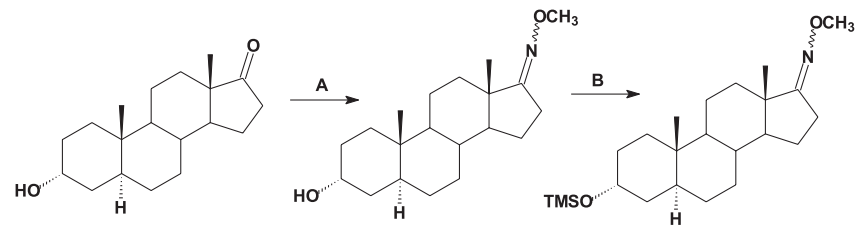

Figura 5. Derivatização da androsterona em duas etapas. Formação da metoxima na posição 17(A), seguida da silanização na posição 3(B)

Baillie e Brooks ${ }^{16}$ avaliaram uma série de derivados para CT endógenos como o 11-desoxicortisol (Figura 6), comparando-os com derivados MO-TMS. Apesar da formação dos produtos propostos, razões como baixos rendimentos e suscetibilidade à hidrólise levaram à não aplicação de tais derivados na análise de CT.

Uma nova abordagem foi introduzida por Chambaz e colaboradores $^{19}$, quando da proposta de silanização por diferentes reagentes derivatizantes via catálise básica, formando derivados TMS-enolTMS (Figura 7).

Os melhores resultados foram obtidos através do uso de piperidina e acetato (de sódio ou potássio) como catalisador. Nessas condições, observou-se a formação de uma série de derivados de CT com total

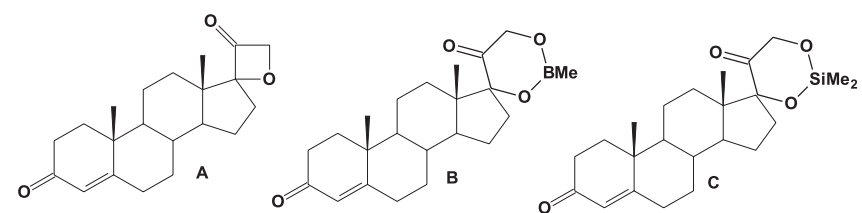

Figura 6. Derivados do 11-desoxicortisol. (A) 17,21-desidro, (B) metilboronatos, $(C)$ dimetilsiliconídeos

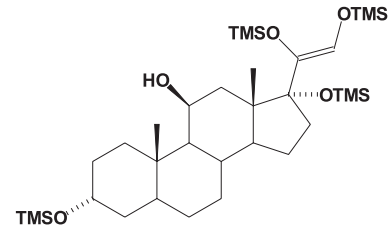

Figura 7. Derivado TMS-enol-TMS do tetraidrocortisol relatado por Chambaz ${ }^{19}$

silanização da cadeia lateral em C17. Grupos hidroxila e cetona na posição 11 não apresentaram reação. CT sintéticos com dupla insaturação no anel A (1,4-dieno) como a triancinolona e a dexametasona não foram investigados. Além disso, os autores discutiram apenas os dados obtidos com acetato de sódio como catalisador.

A silanização de CT também foi alvo da atenção de $\operatorname{Simpson}^{20}$ através da avaliação de CT sintéticos como a triancinolona, betametasona e prednisolona em músculos de rato, utilizando uma mistura de derivatização contendo N-N-bis-trimetilsililacetamida (BSA), N-trimetilsililimidazol (TSIM) e trimetilclorosilano (TMCS) à temperatura ambiente, durante toda a noite.

Observou-se a silanização total dos grupos hidroxila e cetona, inclusive em 1,4-dieno-CT, ao contrário do observado por Chambaz. Além de demonstrar a possibilidade de formação de um único derivado TMS-enol-TMS, sem proteção prévia das carbonilas cetônicas através de metoximas, tal resultado sugere a ocorrência de mecanismos distintos de catálise ácida (Simpson) e básica (Chambaz).

Apesar do resultado de Simpson, derivados MO-TMS foram exaustivamente utilizados nas décadas de 1970 e 1980. Segundo Pfaffenberger e Horning, derivados MO-TMS apresentavam os melhores resultados em análises quantitativas e qualitativas de metabólitos de esteróides, uma vez que previnem a enolização de cetonas através da proteção pelo grupo metoxima. As principais vantagens destacadas são a estabilidade térmica, a volatilidade e a impossibilidade de desidratação com perda de simetria do pico cromatográfico. Porém, nada foi mencionado a respeito da formação dos isômeros sin e anti.

Apesar da possibilidade de formação de isômeros geométricos, derivados MO-TMS foram empregados por Leunissen e Thijsen ${ }^{22}$ em análises quantitativas e avaliação do perfil urinário de esteróides, por Rodchenkov e colaboradores na determinação de metilprednisonola ${ }^{23}$, na elucidação do metabolismo de CT sintéticos como a fluorometolona ${ }^{24}$, betametasona $^{25}$, dexametasona e triancinolona ${ }^{26}$ (Figura 8), e na caracterização da prednisona, prednisolona, e metabólitos ${ }^{27}$.

Entretanto, analitos com substituição na posição 16 apresentam a posição 17 extremamente impedida, o que obriga a realização de reações por até $9 \mathrm{~h}^{26}$. Tal fato não chega a ser um inconveniente significativo em estudos de elucidação de metabolismo de um fármaco. Entretanto, o excessivo tempo de reação impossibilita a utilização de tal derivado como estratégia de triagem no escopo do controle de dopagem. Apesar do exposto, MO-TMS derivados têm sido reportados na monitoração de CT endógenos como nos trabalhos de Yap e colaboradores ${ }^{29,30}$. 
<smiles>COC/C(=N\OC)[C@@]1(OC)CCC2C3C[C@H](C)C4=CC(=N[O+])C=CC4(C)C3C(O[C@@H](C)O)CC21C</smiles>
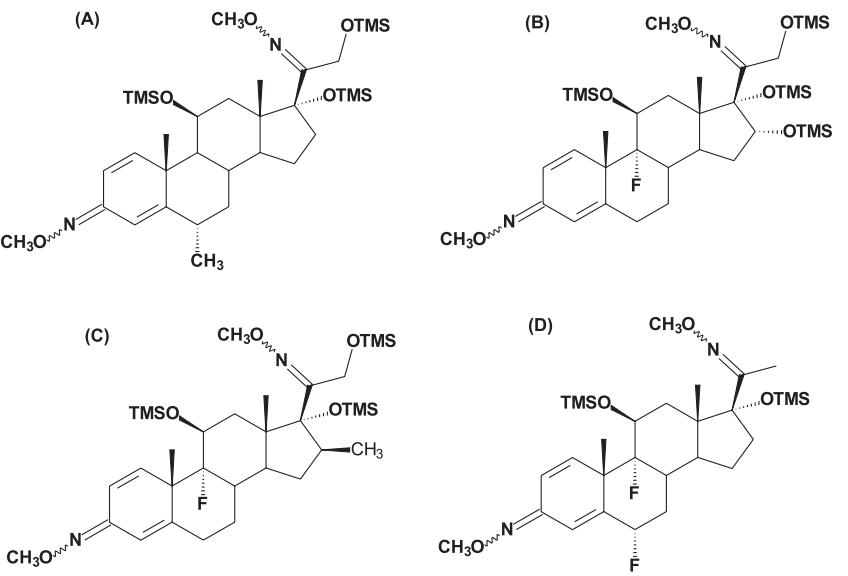

Figura 8. Derivados MO-TMS. (A) diMO-trisTMS-metilprednisolona, (B) diMO-tetraTMS-triancinolona, (C) diMO-trisTMS-betametasona e (D) diMO-diTMS-fluorometolona

A falta de um procedimento eficiente na derivatização dos CT tem levado pesquisadores da área a evitar a CG na análise de CT optando, por exemplo, pela CLAE-EM-EM com a finalidade de evitar a etapa de derivatização. Porém, em um recente trabalho, Bailloux e colaboradore ${ }^{31}$ propuseram a adoção de uma etapa de derivatização antes da análise por CLAE-EM-EM, visando diminuir os limites de detecção e reduzir alguns problemas inerentes à análise.

Por outro lado, outros esteróides, principalmente aqueles com atividade androgênica, têm sido extensivamente estudados por Schänzer e Donike ${ }^{32}$. Somente após um trabalho exaustivo de síntese do reagente de silanização metil-N-trimetilsililtrifluoroacetamida (MSTFA, Schänzer e Donike ${ }^{32}$ ) e da escolha do catalisador apropriado ( $\mathrm{NH}_{4} \mathrm{I}$, trimetiliodosilano), tais esteróides foram eficientemente derivatizados. A enolização total de carbonilas cetônicas na posição 3 foi então explorada através do uso do catalisador trimetiliodossilano (TMSI) na presença de MSTFA. Mais tarde, Opfermann e Schänzer ${ }^{33}$ empregaram uma mistura de silanização que envolve o uso de MSTFA, iodeto de amônio, e eritriol. Posteriormente, o 2-mercaptoetanol substituiu o eritriol por eliminar os problemas de polimerização e conseqüentes interferências observadas na análise. $\mathrm{O}$ mecanismo de catálise ácida está baseado na produção de ácido iodídrico in $s i u^{34}$. Até o presente momento, tal mistura não foi avaliada para análise de CT.

Como a maioria dos estudos de derivatização com CT foram conduzidos empregando o reagente N,O-bis-trimetilsililtrifluoroacetamida (o MSTFA só foi sintetizado em 1969), e o iodeto usado por Opfermann e Schänzer ${ }^{33}$ na derivatização de esteróides androgênicos induz uma via reacional por catálise ácida, o objetivo do presente trabalho foi avaliar o emprego desse procedimento na derivatização de CT. Para tal, comparou-se, para diversos CT endógenos e sintéticos, os resultados obtidos com a mistura MSTFA$\mathrm{NH}_{4}$ I-2-mercaptoetanol (catálise ácida), com os obtidos pelo método descrito por Chambaz (catálise básica) ${ }^{17}$. Comparou-se ainda o desempenho de derivados TMS-enol-TMS com os derivados da metoxima, seguido de silanização (duas etapas).

\section{PARTE EXPERIMENTAL}

\section{Reagentes}

Todos os padrões de CT foram obtidos da Steraloids (NewPort, EUA). N-metil-N-trimetilsililtrifluoroacetamida (MSTFA), Ntrimetilsililimidazol (TSIM), N,O-bis-trimetilsililtrifluoroacetamida
(BSTFA), iodeto de amônio, 2-mercaptoetanol, hidrocloreto de metoxiamina e hidrocarbonetos padrões para o cálculo dos índices de retenção foram obtidos da Sigma (St. Louis, EUA). Piperidina foi obtida da Aldrich (Milwaukee, EUA). Piridina foi obtida da Vetec (Rio de Janeiro, BR). Acetato de sódio foi adquirido da Merck (Rio de Janeiro, BR).

\section{Condições de análise no CG-EM}

As análises foram realizadas em um sistema cromatográfico Agilent 6890 Series, equipado com um injetor automático 7683 com controle eletrônico de pressão e interfaciado com um detector de massas Agilent 5973. Temperaturas de operação do detector: linha de transferência, $280{ }^{\circ} \mathrm{C}$; fonte iônica, $230{ }^{\circ} \mathrm{C}$; interface, $280{ }^{\circ} \mathrm{C}$; quadrupolo, $150{ }^{\circ} \mathrm{C}$. Detecção por monitoramento seletivo de íons (SIM) com um tempo de varredura de 20 milisegundos. Ionização por impacto de elétrons a $75 \mathrm{eV}$. Condições de operação do cromatógrafo: injetor, $280{ }^{\circ} \mathrm{C}$; coluna, $180^{\circ} \mathrm{C}$ (temperatura inicial, $0 \mathrm{~min}$ ); seguido de um gradiente de $3,0^{\circ} \mathrm{C} / \mathrm{min}$ até $229^{\circ} \mathrm{C} / \mathrm{min}$ (0 min) e $40{ }^{\circ} \mathrm{C} / \mathrm{min}$ até a temperatura final de $310{ }^{\circ} \mathrm{C}$ (5,0 min); fluxo total, 18,4 mL/min; pressão, 16,0 psi; velocidade linear média, $38 \mathrm{~cm} / \mathrm{s} ; 1 \mu \mathrm{L}$ de amostra injetada com divisão de fluxo na razão de 1:10. Uma coluna capilar de sílica fundida HP-1 (17,0 m x 0,2 mm x $0,11 \mu \mathrm{m}$ de espessura de filme) foi utilizada.

\section{Condições de derivatização}

A formação dos derivados TMS-enol-TMS por catálise ácida foi avaliada utilizando solução de MSTFA-NH 4 I-2-mercaptoetanol (1000:2:6, v:m:v) a $60^{\circ} \mathrm{C}$ por $20 \mathrm{~min}$. Os derivados MO-TMS foram obtidos pela reação dos CT com uma solução de metoximapiridina $\left(8 \%\right.$ ) a $60^{\circ} \mathrm{C}$ por $30 \mathrm{~min}$. A piridina foi removida sob fluxo de $\mathrm{N}_{2}$ e a silanização foi conduzida com uma mistura MSTFA-TSIM (2\%) a $70{ }^{\circ} \mathrm{C}$ por $15 \mathrm{~min}$. A formação dos derivados TMS-enolTMS por catálise básica foi avaliada utilizando solução de BSTFApiperidina $(2: 1)$ a $60{ }^{\circ} \mathrm{C}$ por $30 \mathrm{~min}$.

\section{Cálculo de índice de Kováts}

Os valores do índice de Kováts foram calculados a partir da análise de uma mistura de hidrocarbonetos lineares (n-alcanos entre $\mathrm{C}_{8}$ $-\mathrm{C}_{36}$ ), utilizando-se as mesmas condições de análise de CG-EM descritas acima, através da equação 1 . Todos ao analitos eluiram durante o gradiente de temperatura.

$$
I=100 n+100 n\left(\frac{t_{R x}-t_{R z}}{t_{R(z+1)}-t_{R z}}\right)
$$

Equação 1: Cálculo do índice de Kováts. n é o número de carbonos do n-alcano com o tempo de retenção $\left(\mathrm{t}_{\mathrm{Rz}}\right)$ imediatamente anterior ao padrão, e $t_{\mathrm{Rz}_{\mathrm{z}+1}}$ o tempo de retenção do $n$-alcano localizado imediatamente após o padrão avaliado

Cálculo do fator de resposta

No cálculo do fator de resposta dos derivados TMS-enol-TMS e MO-TMS o $9 \alpha$-fluoro-17 $\alpha$-metil-4-androsteno-3 $\alpha, 6 \beta, 11 \beta, 17 \beta$-tetrol $\left(\right.$ Fluoxy $-M_{1}$ ) foi utilizado como padrão de referência (Tabelas 1 e 2).

\section{RESULTADOS E DISCUSSÃO}

\section{Formação de derivados TMS-enol-TMS via catálise básica}

A reprodução do experimento de $\mathrm{Chambaz}^{19}$, realizada pelo LAB DOP utilizando piperidina como catalisador, mostrou que cetonas 
Tabela 1. Resultados cromatográficos, espectrométricos e fatores de resposta (FR) de derivados TMS-enol-TMS de CT

\begin{tabular}{|c|c|c|c|c|c|}
\hline Glicocorticosteróides & Derivados & Íons Característicos m/z (int. rel. \%) & F.R. & $\mathrm{t}_{\mathrm{R}}(\mathrm{min})$ & I.K. \\
\hline Tetraidrocortisona & Tetrakis - TMS (- 18) & $634(14) ; 619(5) ; 529(7)$ & 0,62 & 18,13 & 3075,73 \\
\hline Tetraidrocortisol & Tetrakis - TMS (- 18) & $636(60) ; 546(3) ; 282(6)$ & 4,01 & 18,33 & 3126,71 \\
\hline Prednisona & Tetrakis - TMS (- 18) & $628(91) ; 613(4) ; 557$ (6) & 1,12 & 18,40 & 3152,82 \\
\hline 11-Desoxicortisol & Tris - TMS (- 18) & $544(51) ; 529(3) ; 272(4)$ & 4,49 & 18,43 & 3161,72 \\
\hline Desoxicorticosterona & Tris - TMS (- 18) & 546 (50); 301(15); 230 (17) & 4,18 & 18,48 & 3171,51 \\
\hline Cortisona & Tetrakis - TMS (- 18) & 630 (9); 615 (22); 147 (15) & 1,02 & 18,66 & 3219,83 \\
\hline Beclometasona & Tetrakis - TMS (- 18) & $570(35) ; 555$ (4); $296(15)$ & 0,56 & 18,68 & 3233,80 \\
\hline Betametasona & Tetrakis - TMS (- 18) & 662 (11); $456(6) ; 206(8)$ & 1,91 & 18,77 & 3251,12 \\
\hline Dexametasona & Tetrakis - TMS (- 18) & 662(11); 456(6); $206(8)$ & 2,25 & 18,77 & 3251,12 \\
\hline Prednisolona & Tetrakis - TMS (- 18) & $630(14) ; 615(2) ; 191$ (14) & 0,70 & 18,90 & 3295,25 \\
\hline Corticosterona & Tetrakis - TMS (- 18) & $634(20) ; 283(3) ; 230$ (19) & 3,55 & 18,95 & 3310,38 \\
\hline Cortisol & Tetrakis - TMS (- 18) & $632(100) ; 316(5) ; 193$ (4) & 3,42 & 18,96 & 3313,52 \\
\hline Isoflupredona & Tetrakis - TMS (- 18) & 648 (12); 442 (7); 206 (9) & 0,20 & 18,99 & 3322,96 \\
\hline $6 \alpha$-metilprednisolona & Tetrakis - TMS (- 18) & $644(7) ; 424(6) ; 147(10)$ & 1,18 & 19,02 & 3332,39 \\
\hline Triancinolona & Pentakis - TMS (- 18) & 738 (4); $736(10) ; 147$ (9) & 0,13 & 19,18 & 3382,70 \\
\hline $6 \beta$-hidroxicortisol & Pentakis - TMS (- 18) & $720(31) ; 360(7) ; 317$ (4) & 2,97 & 19,38 & 3418,98 \\
\hline
\end{tabular}

Os íons em negrito foram utilizados no cálculo dos fatores de resposta. O padrão utilizado foi a fluoxi-M1 $\left(\mathrm{t}_{\mathrm{R}}=6,77 \mathrm{~min} ; \mathrm{m} / \mathrm{z}=642\right)$. Cálculo do índice de Kováts (I.K.) ver experimental.

Tabela 2. Resultados cromatográficos, espectrométricos e fatores de resposta (FR) de derivados MO-TMS de CT

\begin{tabular}{|c|c|c|c|c|c|}
\hline Glicocorticosteróides & Derivados & Íons Característicos m/z (int. rel. \%) & F.R. & $\mathrm{t}_{\mathrm{R}}(\min )$ & I.K. \\
\hline Tetraidrocortisona & MO - Tris TMS & 609 (23); 578 (100); 488 (64) & 1,33 & 17,70 & 2665,85 \\
\hline Tetraidrocortisol & MO - Tetrakis TMS & 653 (100); 562 (64); $472(31)$ & 0,89 & 17,98 & 2724,62 \\
\hline Desoxicorticosterona & Bis MO - TMS & 460 (93); 429 (100); $286(61)$ & 0,35 & 18,07 & 2748,97 \\
\hline 11-Desoxicortisol ${ }^{1}$ & Bis MO - Bis TMS & 548 (17); 517 (100); 427 (18) & 0,48 & 18,24 & 2792,05 \\
\hline 11-Desoxicortisol ${ }^{2}$ & Bis MO - Bis TMS & 548 (16); 517 (100); 427 (22) & 1,12 & 18,27 & 2800,00 \\
\hline Prednisona $^{1}$ & Bis MO - Bis TMS & $560(26) ; 529(37) ; 309(100)$ & 0,06 & 18,56 & 2885,93 \\
\hline Prednisona $^{2}$ & Bis $\mathrm{MO}-\mathrm{Bis}$ TMS & $\mathbf{5 6 0}(20) ; 529(31) ; 309(100)$ & 0,07 & 18,58 & 2893,11 \\
\hline Cortisona $^{1}$ & Bis MO - Bis TMS & $562(30) ; 531(100) ; 441(38)$ & 0,15 & 18,60 & 2897,90 \\
\hline Cortisona $^{2}$ & Bis MO - Bis TMS & $562(31) ; 531(100) ; 441(38)$ & 0,49 & 18,64 & 2910,03 \\
\hline Corticosterona $^{1}$ & Bis MO - Bis TMS & 548 (100); 517 (89); 427 (52) & 0,22 & 18,67 & 2920,06 \\
\hline Corticosterona $^{2}$ & Bis MO - Bis TMS & 548 (100); 517 (89); 427 (49) & $-*$ & 18,70 & 2927,59 \\
\hline Prednisolona & Bis MO - Tris TMS & $634(24) ; 603(100) ; 262(61)$ & 0,35 & 18,83 & 2969,91 \\
\hline Cortisol $^{1}$ & Bis MO - Tris TMS & $636(19) ; 605(100) ; 515$ (36) & 0,48 & 18,83 & 2969,91 \\
\hline $6 \alpha-$-metilprednisolone ${ }^{1}$ & Bis MO - Tris TMS & $648(18) ; 617(100) ; 276(18)$ & 0,20 & 18,84 & 2972,41 \\
\hline Cortisol $^{2}$ & Bis MO - Tris TMS & 636 (19); 605 (100); 515 (30) & 0,72 & 18,86 & 2979,94 \\
\hline $6 \beta$-hidroxicortisol ${ }^{1}$ & Bis MO - Tetrakis TMS & 725 (23); 694 (100); 603 (53) & 0,08 & 18,88 & 2984,95 \\
\hline $6 \alpha-$ metilprednisolona ${ }^{2}$ & Bis MO - Tris TMS & $648(13) ; 617(100) ; 276(91)$ & 0,21 & 18,92 & 2997,49 \\
\hline $6 \beta$-hidroxicortisol ${ }^{2}$ & Bis MO - Tetrakis TMS & 725 (28); $694(100) ; 603$ (71) & 0,19 & 18,98 & 3000,00 \\
\hline Isoflupredona & Bis MO - Tris TMS & $652(4) ; 621(8) ; 350(\mathbf{1 0 0})$ & 0,79 & 19,04 & 3000,00 \\
\hline
\end{tabular}

Os íons em negrito foram utilizados no cálculo dos fatores de resposta. O padrão utilizado foi a fluoxi-M1 ( $\left.\mathrm{t}_{\mathrm{R}}=16,77 \mathrm{~min} ; \mathrm{m} / \mathrm{z}=642\right)$. Os índices 1 e 2 representam as formas isoméricas anti/sin dos derivados MO - TMS. Cálculo do índice de Kováts (I.K.) ver experimental. * Integrado juntamente com o outro isômero.

na posição 3-ceto-4-eno (conjugadas) apresentam enolização parcial, formando dois derivados (Figura 9). A formação de múltiplos derivados é um inconveniente analítico na detecção de CT endógenos como o cortisol, cortisona e 11-desoxicortisol.

Como esperado 1,4-dieno-CT não apresentam reação no anel A. Neste estudo, observa-se a formação da dexametasona-tris-TMS com total silanização do anel $\mathrm{D}$, inclusive na posição 17 , consideravelmente impedida pelo grupo metila na posição 16 (Figura 10). Com relação aos catalisadores utilizados, o autor menciona as vantagens da piperidina sobre os sais de acetato (mesma eficiência, porém maior volatilidade e forma líquida). Foi observado, porém, que a piperidina está sujeita a degradação pela exposição à atmosfera, o que preconiza a adoção de controles de derivatização e cuidados na manipulação, além de purificações periódicas, indesejáveis no contexto de rotina.

\section{Formação de derivados TMS-enol-TMS via catálise ácida}

O procedimento de formação de TMS-enol-TMS por catálise ácida com $\mathrm{NH}_{4} \mathrm{I}$, apresenta maior praticidade se comparado ao uso de metoxiamina, produzindo, com alto rendimento e em um tempo de reação menor, apenas um derivado para cada CT avaliado (Tabela 1). Apesar do grupo hidroxila no carbono 17 não ser silanizado (a perda de água é o processo mais significativo no espectro de massas, 


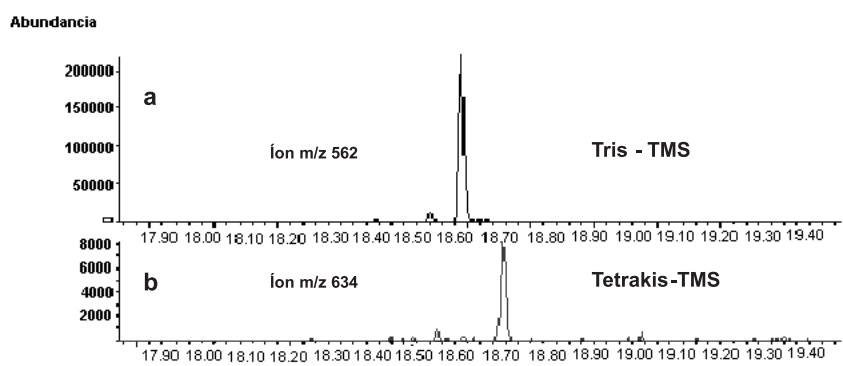

Figura 9. Resultados da análise de CG-EM obtidos por varredura linear dos derivados do 11-desoxicortisol formados pela reação com BSTFA catalisada por piperidina. (a) Cromatograma do íon $\mathrm{m} / \mathrm{z} 562$ do derivado enol-Tris-TMS. (b) Cromatograma do íon $\mathrm{m} / \mathrm{z}, 634$ do derivado enol-Tetrakis$T M S$

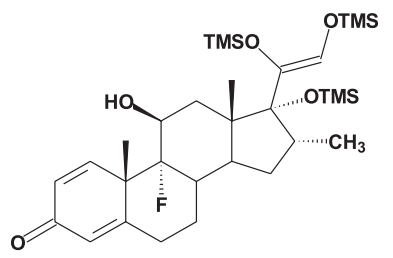

Figura 10. Dexametasona enol-tris-TMS

Tabela 1 e Figura 11), os picos são simétricos e os resultados reprodutíveis.Nos $\mathrm{CT}$ sintéticos e endógenos com grupos cetona no anel A, observou-se enolização total das carbonilas nas posições $3 \mathrm{e}$ $20^{35}$. A enolização do anel A em 3-ceto-4-eno conjugados (CT do grupo 2), via catálise ácida com MSTFA, iodeto de amônio, e 2mercaptoetanol, com formação do 3,5-dienol é esquematizada na Figura 12.

Parâmetros como perfil cromatográfico, fator de resposta e índice de Kováts foram avaliados para vários CT endógenos e sintéticos
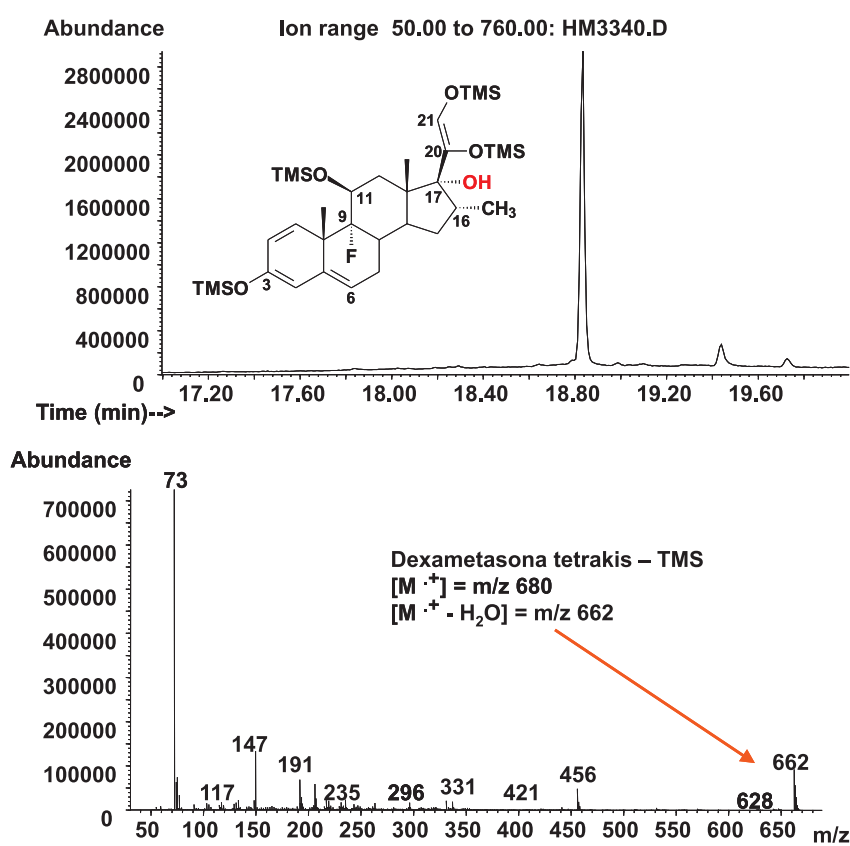

Figura 11. Cromatograma de ions totais e espectro de massas da dexametasona-Tetrakis-TMS quanto à formação de derivados TMS-enol-TMS (Tabela 1). Os resultados encontrados para os derivados MO-TMS são relatados com o objetivo de servir como parâmetro de comparação (Tabela 2).

$\mathrm{Na}$ análise dos resultados do derivado MO-TMS para a corticosterona, apesar da separação visual dos picos dos isômeros 3anti/sin, a área referente aos derivados foi integrada como um único pico.

Para cada CT avaliado, os derivados TMS-enol-TMS apresentaram um fator de resposta superior aos derivados MO-TMS correspondentes, excetuando-se para a tetraidrocortisona e a isoflupredona (Tabelas 1 e 2). Nas condições cromatográficas adotadas, a análise dos índices de Kováts apresentou coeluição entre CT sintéticos e endógenos para alguns derivados MO-TMS, como observado entre a prednisolona e um dos isômeros geométricos do cortisol (Figura 13), para o 6 $\beta$-hidroxicortisol (um metabólito do cortisol) e a isoflupredona. Tal fato pode se constituir em uma desvantagem con-

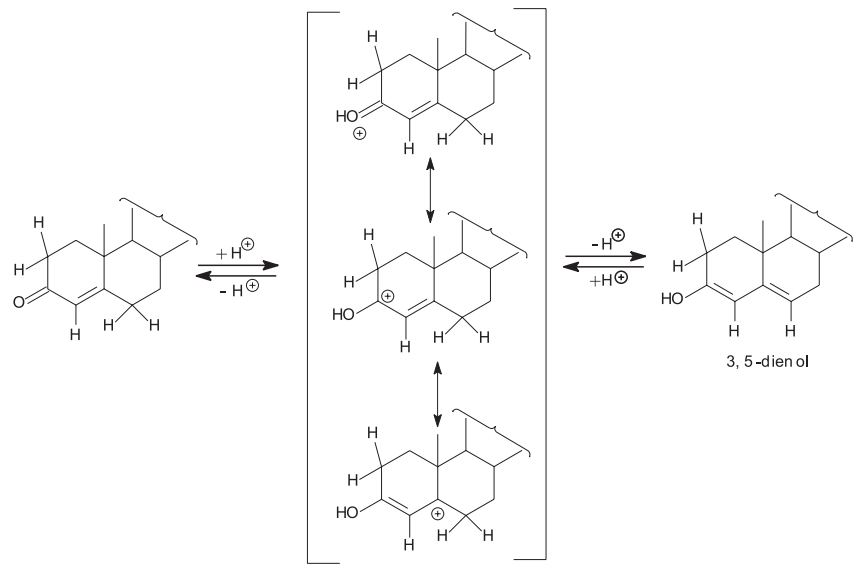

Figura 12. Proposta de formação do 3,5-dienol em CT do grupo 2 por catálise ácida através da reação com MSTFA, $\mathrm{NH}_{4}$ I e 2-mercaptoetanol

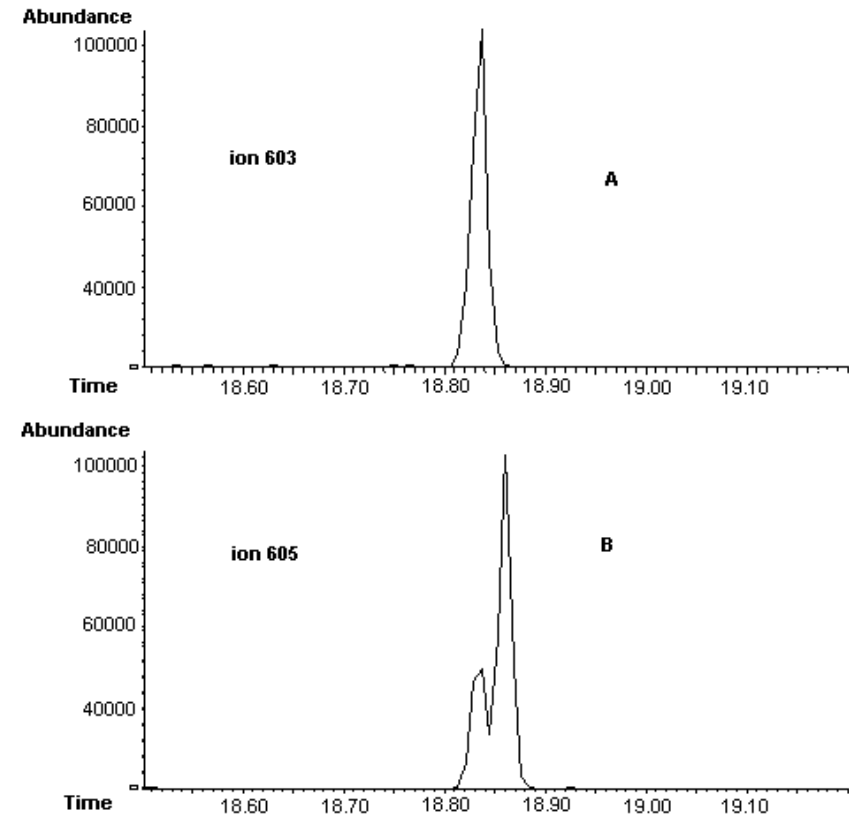

Figura 13. Resultados da análise de CG-EM por varredura linear. (A) Cromatograma do íons $\mathrm{m} / \mathrm{z} 603$ da prednisona bis-MO-tris-TMS. (B) Cromatograma do íon $\mathrm{m} / \mathrm{z}, 605$ do cortisol bis-MO-tris-TMS 
siderável na detecção de CT sintéticos, uma vez que se espera que os CT endógenos sejam excretados em alta concentração. Por outro lado, dentre os derivados TMS-enol-TMS, apenas os epímeros dexametasona e betametasona apresentam coeluição. Nenhuma coeluição foi observada entre os derivados TMS-enol-TMS sintéticos e endógenos.

\section{CONCLUSÕES}

Até o presente momento, não há na literatura um procedimento eficiente para a derivatização dos glicocorticóides (CT). Embora a formação de MO-TMS seja a estratégia mais freqüentemente empregada, tem-se observado a tendência de substituir a CG por técnicas como a CLAE-EM-EM com a finalidade de evitar a etapa de derivatização.

Os derivados TMS-enol-TMS obtidos através da silanização via catálise ácida com $\mathrm{NH}_{4} \mathrm{I}$, por outro lado, mostram comportamento cromatográfico nitidamente superior aos correspondentes derivados metoxima trimetilsilila. Isto se deve, entre outros fatores, ao fato de que a formação de derivados MO-TMS em compostos que apresentam impedimento estérico no anel D só é possível através de um longo tempo e condições severas de reação. Portanto, tal procedimento torna-se inviável no contexto de análises de triagem. Em adição, para derivados MO-TMS, alguns CT exógenos mostram coeluição com CT endógenos (excretados em altas quantidades), tornando difícil a avaliação do perfil de endógenos e o diagnóstico do abuso de CT exógenos. Além disto, o processo de derivatização envolvendo a mistura MSTFA-NH -2 -mercaptoetanol mostrou-se eficiente para todos os CT considerados. Estudos posteriores serão realizados em urina para avaliar a viabilidade deste procedimento na etapa de triagem de CT em baixas concentrações no controle de dopagem.

\section{REFERÊNCIAS}

1. Haynes Jr., R. C; Murad, F. Em As Bases Farmacológicas da Terapêutica; Goodman, L.S.; Gilman, A.G., eds.; Guanabara Koogan: Rio de Janeiro, 1987, p. 943.

2. Goth, A.; Shore, P. A.; Farmacologia Médica, Guanabara Koogan: Rio de Janeiro, 1981, p. 393.

3. Venturelli, E.; Cavarelli, A.; Secreto, G.; J. Chromatogr., B 1995, 671, 363.

4. Popot, M. A.; Garcia, P.; Bonnaire, Y. Em Recent Advances in Doping Analysis (5); Schänzer, W.; Geyer, H.; Gotzmann, A.; Mareck-Engelke, U., eds.; Sport und Buch Straub: Köln, 1997, p. 185.

5. Stanley, S. M.; Wilhelmi, B. S.; Rodgers, J. P.; J. Chromatogr. 1993, 614, 77.

6. Kim, Y.; Kim, T.; Lee, W.; Rapid Commun. Mass Spectrom. 1997, 11, 863.
7. Her, G. R.; Watson, J. T.; Anal. Biochem. 1985, 151, 292.

8. The OMAC 1999. Olympic Movement Anti-doping Code. Appendix A. 4.

9. Catlin, D. H.; Murray, T. H.; J. Am. Med. Assoc. 1996, 276, 231.

10. Jusko, W. J.; Pyszczynski, N. A.; Bushway, M. S.; D’Ambrosio, R.; Mis, S. M.; J. Chromatogr., B 1994, 658, 47.

11. Barbarulo, M. V.; Buiarelli, F.; Ciardi, M.; Giarrusso, A.; Rosati, F. Em $10^{\text {th }}$ Cologne Workshop on Dope Analysis; Proccedings.; Donike, M.; Geyer, H.; Gotzmann, A.; Mareck-Engelke, U.; Rauth, S., eds.; Sport and Buch Straub: Cologne, 1992, p. 249.

12. Creaser, C. S.; Feely, S. J.; Houghton, E.; Seymour, M.; J. Chromatogr., A 1998, 794, 37

13. Savu, S. R.; Silvestro, L.; Haag, A.; Sörgel, F.; J. Mass Spectrom. 1996, 31,1351 .

14. Delahaut, Ph.; Jacquemin, P.; Colemonts, Y.; Dubois, M.; De Graeve, J.; Deluyker, H.; Chromatography 1997, 696, 203.

15. Ishibashi, M.; Takayama, H.; Nakagawa, Y.; Noriaki, H.; Chem. Pharm. Bull. 1988, 36, 845 .

16. Baillie, T. A.; Brooks, C. J. W.; Anal. Chem. 1972, 44, 30.

17. Chambaz, E. M.; Defaye, G.; Madani, C.; Anal. Chem. 1973, 45, 1090.

18. Fales, H. M.; Luukkainen, T.; Anal. Chem. 1965, 37, 955.

19. Chambaz, E. M.; Horning, E. C.; Anal. Biochem. 1969, 30, 7.

20. Simpson, P. M.; J. Chromatogr. 1973, 77, 161.

21. Pfaffenberger, C. D.; Horning, E. C.; High Resol. Biomed. Gas Chromatog. 1975, 112, 581 .

22. Leunissen, W. J.; Thijsen, J. H. H.; J. Chromatogr. 1978, 146, 365.

23. Rodchenkov, G. M.; Uralets, V. P.; Semenov, V.; J. Chromatogr. 1987, 423, 15.

24. Rodchenkov, G. M.; Uralets, V. P.; Semenov, V. A.; J. Chromatogr. 1988 , $426,399$.

25. Rodchenkov, G. M.; Uralets, V. P.; Semenov, V. A.; J. Chromatogr. 1988, 432, 283.

26. Rodchenkov, G. M.; Uralets, V. P.; Semenov, V. A.; Leclercq, P. A.; J. High Resolut. Chromatogr. Chromatogr. Commun. 1988, 11, 283.

27. Rodchenkov, G. M.; Vedenin, A. N.; Uralets, V. P.; Semenov, V. A.; J. Chromatogr. 1991, 565, 45.

28. Horning, M. G.; Moss, A. M.; Horning, E. C.; Anal. Biochem. 1968, 22, 284

29. Yap, B. K.; Johnston, G. A.; Kazlauskas, R.; J. Chromatogr. 1992, 573, 183.

30. Yap, B.; Kazlauskas, R. Em Recent Advances in Doping Analysis (7); Schänzer, W.; Geyer, H.; Gotzmann, A.; Mareck-Engelke, U., eds.; Sport and Buch Straub: Cologne, 1999, p. 85.

31. Bailloux, I.; De Barros, S.; Lahaussois, A.; Mathurin, J. C.; De Ceaurriz, J.; Ref. 30, p. 203.

32. Schänzer, W.; Donike, M.; Anal. Chim. Acta, 1993, 275, 23.

33. Opfermann, G.; Schänzer, W. Em Recent Advances in Doping Analysis (4); Schänzer, W.; Geyer, W.; Gotzmann, A.; Mareck-Engelke, U., eds.; Sport and Buch Straub: Cologne, 1996, p. 247.

34. Van der Kerkhof, D. H.; Zwikker, J. W.; Kaats-Richters, V. E. M.; Bouwman, K.; van Ooijen; de Boer, D.; Maes, R. A. A.; Ref. 30, p. 129.

35. Pereira, H.M.G.; Marques, M.A,S.; Cardoso, J.N.; de Aquino Neto, F. R. Em Recent Advances in Doping Analysis (9); Schänzer, W.; Geyer, H.; Gotzmann, A.; Mareck-Engelke, U., eds.; Sport and Buch Strauß: Cologne, 2001, p. 203. 